\title{
STR Analysis of Human DNA Samples After Dry-State Ambient Temperature Storage in GenPlates
}

\author{
Richard I. Somiari ${ }^{*}, 1$, Ebenezer Adebiyi ${ }^{\sharp 1,}$, Livinus Ukachukwu ${ }^{\#, 1}$, Igbe I. Mba ${ }^{\#, 1}$, Friday A. Anthony ${ }^{\sharp, 1}$, \\ Anthony O. Ogundele ${ }^{\#, 1}$, Innocent Onuaha ${ }^{\#, 1}$, Marissa Brainard ${ }^{1}$, Susan Lubert ${ }^{2}$, Caroline Larson ${ }^{2}$, \\ Stephen Russell ${ }^{1}$, Narayanaswamy Bharathan ${ }^{3}$ and Stella B. Somiari ${ }^{2}$ \\ ${ }^{I}$ ITSI - Biosciences, Johnstown, PA, USA \\ ${ }^{2}$ Windber Research Institute, Windber, $P A$, USA \\ ${ }^{3}$ Indiana University of Pennsylvania, Indiana, PA, USA
}

\begin{abstract}
Dry-state room temperature storage of DNA is of interest because it promises to be a relatively more cost effective and environmentally friendly method for transport and storage of DNA. However, this approach must be validated prior to large scale adoption by the scientist that currently store blood samples frozen. In this study, we compared the yield of DNA isolated from GenPlates ${ }^{\mathrm{TM}}$ to that from frozen blood, and verified the quality of the DNA by evaluating the success of genotyping and allelic assignment using the PowerPlex 16 system. Using 24 blood specimens from 6 human subjects, we determined that DNA yield from GenPlates ${ }^{\mathrm{TM}}$ a 96 well microtitre plate based product for transport and storage of DNA in the dry-state and at ambient temperature was $4.9 \%$ more than the yield from frozen blood after 16 months storage. The STR profiles from GenPlates ${ }^{\mathrm{TM}}$ DNA displayed $100 \%$ concordance with the profile from DNA isolated from frozen blood. Our results demonstrate that yield and PowerPlex 16 STR profile of DNA after dry-state ambient temperature storage in GenPlates ${ }^{\mathrm{TM}}$ for 16 months are comparable to DNA from frozen blood and $100 \%$ allelic assignment was achieved using $1 \mathrm{ng}$ of DNA isolated from GenPlates ${ }^{\mathrm{TM}}$.
\end{abstract}

Keywords: GenPlates, Dry-state, DNA yield, PowerPlex 16.

\section{INTRODUCTION}

Forensic DNA analysis is becoming the standard for primary or secondary identification of human remains with high confidence. The introduction of short tandem repeat (STR) loci in paternity testing and forensic identification of human remains has helped increase the value of DNA profiling, and wider adaptation of this approach. Blood remains one of the most important and useful source of DNA for forensic applications and biomedical research because it provides high yield and good quality DNA that is suitable for many downstream applications [1]. The most widely used method for storage and transport of blood is freezing. Unfortunately, handling and transport of blood in the liquid state from the site of collection to the site of analysis and storage involves the risks of leakage and sample degradation due to temperature variation [2]. Maintaining a cold chain is expensive, especially in resource challenged parts of the world where public electricity supply is not reliable and/or expensive. The cost for sample collection and storage increases significantly with increasing number of samples, because of the requirement for space and freezers, if long term storage will be by frozen storage.

\footnotetext{
*Address correspondence to this author at ITSI - Biosciences LLC, 633 Napoleon Street, Johnstown, PA 15901, USA; Tel: +1-814-262-7331; Fax: +1-814-262-7334; E-mail: Richard@itsibio.com

"Present Address: Defence Identification Center, Nigerian Defence Headquarters, Abuja, Nigeria
}

There is growing interest to develop reference DNA banks for multiple applications, including: a) national security operations, b) identification of human remains, c) establishment/confirmation of identity, d) re-association of remains and e) biomedical research. Due to the relatively higher cost associated with liquid-state handling and frozen storage of DNA, it will be desirable to transport and store DNA specimens in the dry-state at ambient temperature. Moreover, dry state transport and storage is environmentally friendly and will allow the collection and maintenance of larger reference DNA collections [2].

Paper (e.g. Guthrie and Whatman cards) has been used for many years for collection, transportation and storage of blood at ambient temperature [3]. After spotting and drying, the paper is typically placed in envelopes or files, stored in cabinets or shelves. Blood collection with paper is attractive compared to tubes because less blood is required, immediate additional processing is not necessary and once dry, the blood is not generally infectious [4,5]. There are many paper-based DNA repositories with several million samples. For example the United States Armed Forces Repository of Specimen Samples for the Identification of Remains (AFRSSIR, http://www.afmes.mil/) now contains over 4.5 million samples spotted on paper.

There are new commercially available products that are developed specifically for dry-state storage of whole-blood and purified nucleic acids [6,7]. GenVault Corp provides GenPlates $^{\mathrm{TM}}$, specifically developed for whole blood DNA collection, transport and dry-state storage at ambient 
temperature. GenPlates ${ }^{\mathrm{TM}}$ contain $6 \mathrm{~mm}$ disc of FTA paper elements placed in each well of a microtitre plate. According to the manufacturer, the FTA paper used is "a macroporous cellulose matrix that is chemically treated to inactivate bacteria and viruses". When a biological sample, especially whole blood is applied, the proprietary chemical lyses the cell releasing DNA which becomes entwined in the fibrous network of the cellulose matrix. This unique presentation of FTA paper in a microtitre plate format has potential for blood sample transport and large scale reference DNA collection because it brings together the strengths of FTA cards and microtitre plates.

In this paper, data is presented for yield and quality of DNA collected in GenPlates and stored at room temperature for up to 16 months. Total DNA isolated per microliter of blood was used as the criterion for yield. Genotyping success was used as the criterion for quality, and this was evaluated with the PowerPlex 16 system (Promega Corporation). The PowerPlex 16 system was chosen because it allows multiplexed Short Tandem Repeat (STR) analysis of 16 loci, consisting of the 13 core Combined DNA Index System (CODIS) loci plus amelogenin, and two pentanucleotide STR loci, Penta D and Penta E [8]. This means that the ability to use the GenPlates"TM blood as "reference" to identify randomly coded donor blood samples can be simultaneously evaluated. Data generated from 6 human donors demonstrates that the amount of DNA recovered from GenPlates ${ }^{\mathrm{TM}}$ are comparable to those from frozen blood after 16 months storage, and that $100 \%$ allelic assignment and identification of 6 human subjects was achieved using 1 $\mathrm{ng}$ of DNA isolated from GenPlates ${ }^{\mathrm{TM}}$.

\section{MATERIALS AND METHODS}

\subsection{Source of Blood Samples}

Approximately $5 \mathrm{ml}$ of blood was collected from 6 male donors of African origin by venipuncture. Blood was collected with a blood DNA collection kit (ITSI-Biosciences, Johnstown, PA) containing a $2 \mathrm{ml}$ BD Vacutainer tube (REF 367842) with EDTA as anticoagulant. After transfer of blood into the vacutainer tube, the tube was inverted about 5 times to mix the blood and reagent, and stored at room temperature for approximately $10 \mathrm{~min}$ before processing.

\subsection{Blood Sample Processing}

The blood samples were assigned codes to protect the privacy of the donors, and to allow for unbiased evaluation of yield and quality. Each sample was aliquoted $(0.5 \mathrm{ml}$ aliquots) into $1.5 \mathrm{ml}$ screw capped tubes and stored at $-80^{\circ} \mathrm{C}$ until needed. Additionally, 10ul of blood was spotted into each well of 24-Region GenPlates ${ }^{\mathrm{TM}}$ (GVN24P(4)-20; GenVault Corporation). The 24-Region plates are designed to hold samples from 24 donors in 4 aliquots. Thus, only 40ul of blood was required from each donor for spotting into 24-Region GenPlates ${ }^{\mathrm{TM}}$. Because the blood from each donor was spotted into 4 wells, this study evaluated a total of 24 specimens (6 donors $\mathrm{x} 4$ aliquots) at each time point. After spotting, the GenPlates ${ }^{\mathrm{TM}}$ were placed in a Field Drying Kit (GVFAP-5; GenVault Corporation) containing a desiccant, and allowed to dry at ambient temperature for $24 \mathrm{~h}$. An adhesive plate seal was applied to the top of each GenPlates $^{\text {TM }}$ before placement in a Desktop Archive (GV2;
GenVault Corporation) for dry-state ambient temperature storage at $23 \pm 1^{\circ} \mathrm{C}$. For this study, one tube of blood was removed from the freezer and 4 elements were punched out of the GenPlates ${ }^{\mathrm{TM}}$ after storage for 2 weeks and 16 months respectively. It is recommended that GenPlates ${ }^{\mathrm{TM}}$ be allowed to dry and stored for at least 2 weeks prior to DNA isolation.

\subsection{Isolation of DNA from frozen and GenPlates ${ }^{\mathrm{TM}}$ Blood}

DNA was isolated from 200 ul of frozen blood using the Qiagen QIAmp DNA Blood Kit according to the recommended protocol (Qiagen Inc, Valencia, CA). DNA was independently isolated from the four elements of GenPlates $^{\mathrm{TM}}$ onto which $10 \mathrm{ul}$ of blood were spotted into each well. DNA isolation was with the GenSolve Kit and recommended protocol (GVR-100, GenVault Carlsbad, CA), and the isolated DNA was purified with the Qiagen QIAmp DNA Mini Kit (Qiagen Inc, Valencia, CA) using the protocol recommended by GenVault Corporation.

\subsection{Quantitation of Isolated DNA}

Total DNA isolated from frozen blood and GenPlates ${ }^{\mathrm{TM}}$ blood were quantified with Quant-iT dsDNA HS Assay Kit using the Qubit 1.27 Fluorometer (Invitrogen Inc, Carlsbad, CA) as recommended by the manufacturers. Briefly, all reagents from the Assay kit were equilibrated to room temperature before processing and $0.5 \mathrm{ml}$ Qubit Assay tubes were used throughout the experiments. The Quant-iT Working solution was prepared by diluting the Quant-iT dsDNA HS reagent 1:200 in the Quant-iT dsDNA HS Buffer. One hundred ninety microliters of Quant-iT working solution was added to a $0.5 \mathrm{ml}$ Qubit Assay tube and $10 \mathrm{ul}$ of a DNA sample was added to the tube and vortexed. This step was repeated for all standards. The tubes were allowed to incubate at room temperature for 2 minutes before reading. The recommended calibration protocol was followed to calibrate the Qubit Fluorometer. The sample concentrations were calculated with the "Calculate Sample Concentration" function on the fluorometer, using $10 \mathrm{ul}$ as the sample size.

\subsection{DNA Amplification by PCR}

All DNA samples were dried down in an Eppendorf Vacufuge and re-suspended with distilled and deionized nuclease-free water to normalize all samples to $0.5 \mathrm{ng} / \mathrm{ul}$. Then $2 \mathrm{ul}$ of each DNA sample containing 1ng of total DNA was transferred to a fresh tube and 17.2ul Nuclease-free water, 2.5ul GoldStar 10x Buffer, 2.5ul PowerPlex 16 10x Primer Pair Mix and 0.8ul AmpliTaq Gold DNA Polymerase were added to obtain a total reaction volume of $25 \mathrm{ul}$. The mixture was briefly centrifuged and the DNA amplified in an Applied Biosystems 9700 thermal cycler using the 10/22 cycling protocol described in the PowerPlex 16 technical manual.

\subsection{Analysis of Amplified DNA}

After amplification the samples were cleaned to remove all unincorporated fluorescent dyes using Multiscreen Plates (Millipore) filled with DNA grade Sephadex G-50 (GE Healthcare, Piscataway, NJ). The cleaned samples were prepared for analysis on the MegaBACE 1000 [9] by adding $2 \mathrm{ul}$ of the cleaned samples to an 8ul mixture of ET550R (GE Healthcare) and $0.1 \%$ Tween 20 (usb). All samples were electrokinetically injected into the MegaBACE capillaries 
for $45 \mathrm{sec}$ at $3 \mathrm{kV}$ and run for $90 \mathrm{mins}$ at $10 \mathrm{kV}$. The raw data (.rsd) files obtained were entered into MegaBACE Fragment Profiler v1.2 (GE Healthcare) for analysis of all 16 allele markers in each well. For every step mentioned above a positive control included in the PowerPlex 16 System kit was processed in parallel.

\subsection{Identification of Donors Using GenPlates ${ }^{\text {TM }}$ DNA as Reference}

To determine if the PowerPlex 16 STR data from DNA isolated from GenPlates ${ }^{\mathrm{TM}}$ can be used to identify 6 hypothetical cases, we compared the profile of frozen blood (FB) against the profile of a "reference" DNA database created with the profile of DNA isolated from GenPlates ${ }^{\mathrm{TM}}$ blood (GB). The six FB samples were randomly coded C032, C413, C471, C593, C621 and C925 and the GB samples used to construct the database were randomly coded R073, R213, R374, R456, R762 and R810.

\section{RESULTS \& DISCUSSION}

The major objectives of this study were to evaluate the suitability of a) collecting and transporting blood in GenPlates and b) the genotyping quality and amount of DNA that can be recovered from GenPlates ${ }^{\mathrm{TM}}$ after storage at room temperature. We used DNA isolated from frozen blood as the benchmark because it is universally accepted that frozen blood is a good source of high quality DNA that is suitable for forensics and other downstream applications. We obtained blood by venipuncture from 6 fully informed and consented males of African origin that ranged in age from 34 to 48 years old at the time of blood collection. Ten micro liters each of blood from every donor was spotted into 4 wells of a 24-region GenPlates ${ }^{\mathrm{TM}}$. Thus, a total of 24 specimens were analyzed for the study. So far we have generated data from frozen blood and GenPlates ${ }^{\mathrm{TM}}$ blood stored for up to 16 months.

\subsection{DNA Yield from GenPlates ${ }^{\mathrm{TM}}$}

As shown in Table 1, total DNA yield from GenPlates ${ }^{\mathrm{TM}}$ averaged $0.087 \mathrm{ng} / \mathrm{ul}$ and $0.081 \mathrm{ng} / \mathrm{ul}$ after 2 weeks and 16 months dry state ambient temperature storage respectively, whereas the yield from frozen blood averaged $0.059 \mathrm{ng} / \mathrm{ul}$ and $0.077 \mathrm{ng} / \mathrm{ul}$ for the same period, respectively. Considering that $0.5-1 \mathrm{ng}$ total DNA is recommended for STR analysis using PowerPlex 16 [8] indicates that sufficient DNA for STR analysis with the PowerPlex 16 was recovered from $6 \mathrm{~mm}$ disc of FTA after 16 months storage.

A comparison of the DNA yield between GenPlates ${ }^{\mathrm{TM}}$ and frozen blood demonstrates that an average of $32.2 \%$ and $4.9 \%$ more DNA was recovered per ul blood from GenPlates $^{\mathrm{TM}}$ compared to frozen blood after two weeks and 16 months, respectively. This finding is significant because it demonstrates that higher amounts of DNA are recoverable from GenPlates ${ }^{\mathrm{TM}}$ per microliter of blood compared to frozen blood. Two recent papers discuss the recovery of purified DNA from commercially available products designed for dry-state ambient temperature storage of purified DNA [6,7]. The average DNA recovered from GenTegra was reported as $99.2 \pm 12.9 \%$ (6) and $127.8 \%$ [7] more yield than from frozen samples. Unfortunately, we cannot make a direct comparison because the above results are for purified DNA and literature search did not reveal any publication with data for whole blood spotted into GenPlates $^{\mathrm{TM}}$ and stored for up to 16 months.

Table 1. DNA Yield from GenPlates and Frozen Blood After Two Weeks and Sixteen Months Storage of 24 Blood Specimens from Six (S1-S6) Human Subjects

\begin{tabular}{|c|c|c|c|c|}
\hline \multirow{2}{*}{ Subject } & \multicolumn{4}{|c|}{ DNA Yield (ng/ul) } \\
\cline { 2 - 5 } & \multicolumn{2}{|c|}{ GenPlates $^{\text {TM }}$ Blood* } & \multicolumn{2}{|c|}{ Frozen Blood $^{*}$} \\
\cline { 2 - 5 } & $\mathbf{2}$ Weeks & $\mathbf{1 6}$ Months $^{*}$ & 2 Weeks & $\mathbf{1 6}_{\text {Months }}$ \\
\hline \hline S1 & 0.0929 & 0.118 & 0.06 & 0.088 \\
\hline S2 & 0.109 & 0.057 & 0.061 & 0.094 \\
\hline S3 & 0.0535 & 0.087 & 0.055 & 0.068 \\
\hline S4 & 0.0724 & 0.103 & 0.06 & 0.074 \\
\hline S5 & 0.112 & 0.061 & 0.064 & 0.069 \\
\hline S6 & 0.0798 & 0.057 & 0.055 & 0.071 \\
\hline Mean & 0.087 & 0.081 & 0.059 & 0.077 \\
\hline SD & 0.022 & 0.026 & 0.004 & 0.011 \\
\hline
\end{tabular}

*GenPlates were stored in the dry state at ambient temperature $\left(23 \pm 1^{\circ} \mathrm{C}\right)$. ${ }^{\#}$ Frozen blood was stored at $-80^{\circ} \mathrm{C}$. Data for GenPlates are the means of DNA from 4 elements for each individual.

\subsection{Genotyping Quality of DNA from GenPlates ${ }^{\mathrm{TM}}$}

The second major objective of the study was to evaluate the quality of the DNA isolated from GenPlates ${ }^{\mathrm{TM}}$. We achieved this objective by evaluating the success of genotyping and allelic assignment using DNA from GenPlates $^{\mathrm{TM}}$. The PowerPlex 16 system was chosen for STR analysis because it: a) covers the 13 core CODIS loci used with the United States national database system, b) allows a single multiplex STR analysis of 16 STR's with minimal amounts of input DNA and c) has been validated for forensics and database genotyping [8, 10]. To avoid/reduce any experimental variations, DNA isolation from GenPlates $^{\mathrm{TM}}$ and frozen samples were performed on the same day, and normalized to $0.5 \mathrm{ng} / \mathrm{ul}$ before amplification by PCR.

The DNA isolated from frozen blood and GenPlates ${ }^{\mathrm{TM}}$ blood from the 6 donors produced complete STR profiles that were above a threshold fixed to 200 Relative Florescent Units (RFU). As shown in Fig. (1, GB) and Fig. (2, FB) the allelic assignment and pattern of peaks for each allele were comparable for GB and FB. The Red traces on each plot represent the internal standard ET55OR, the blue traces represent dye FAM (Panel A), the black traces represent the dye TMR (Panel B) and the green peaks represent dye JOE (Panel C). Although, all 16 loci were detected for the six donors, for FB and GB DNA samples, the signal intensity from GB DNA were apparently lower than those for FB DNA in 14 out of the 16 STR's (Fig. 3). Of significance is the finding that there was $100 \%$ concordance between DNA from $\mathrm{GB}$ and $\mathrm{FB}$ at all loci based on allelic assignment, an indication that quality of DNA from GenPlates ${ }^{\mathrm{TM}}$ is comparable to DNA from frozen blood after up to 16 months ambient temperature storage. 


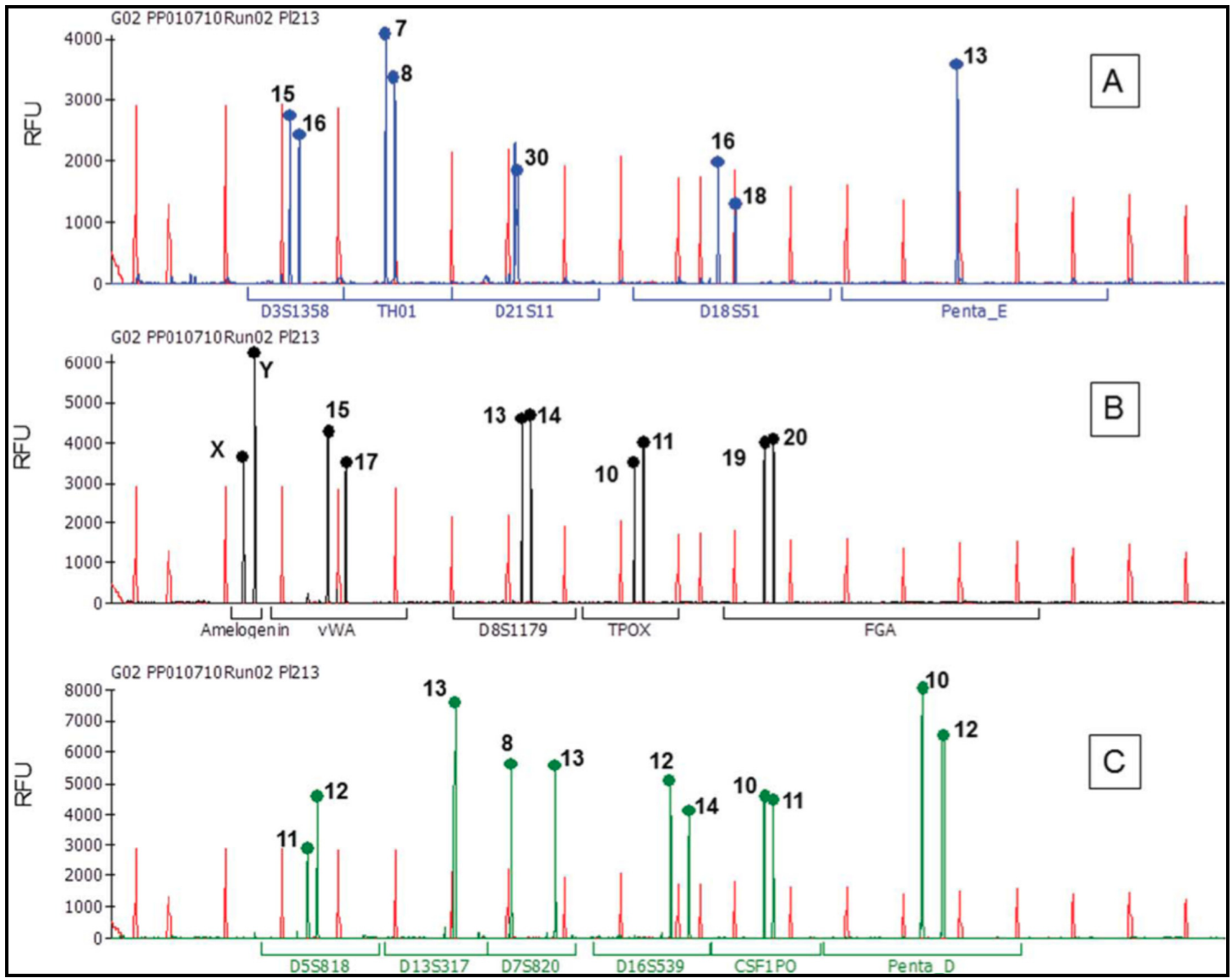

Fig. (1). Representative PowerPlex 16 STR profile of DNA isolated from GenPlate ${ }^{\mathrm{TM}}$ spotted with blood from subject \#213.

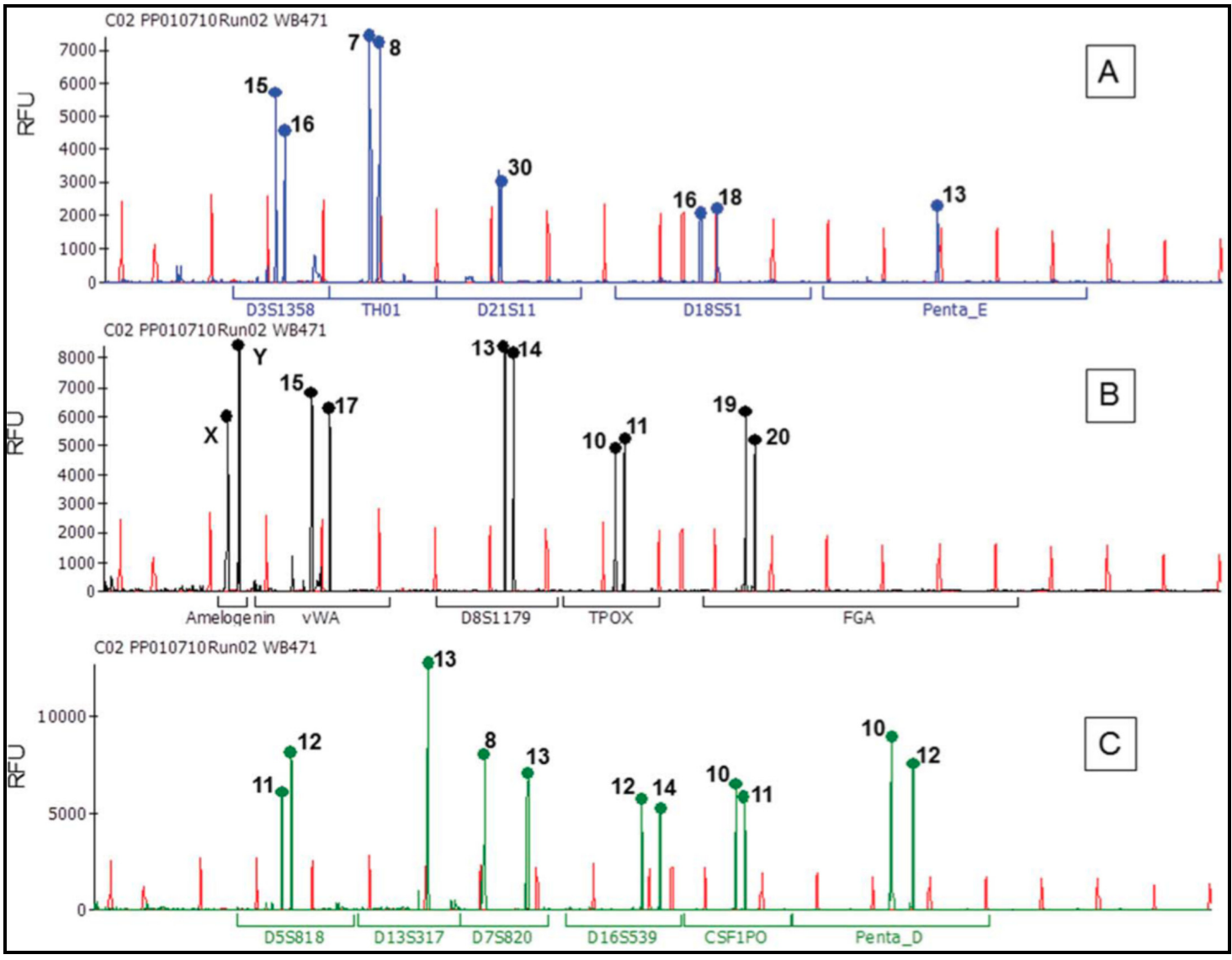

Fig. (2). Representative PowerPlex 16 STR profile of DNA isolated from frozen blood from subject \#471. 


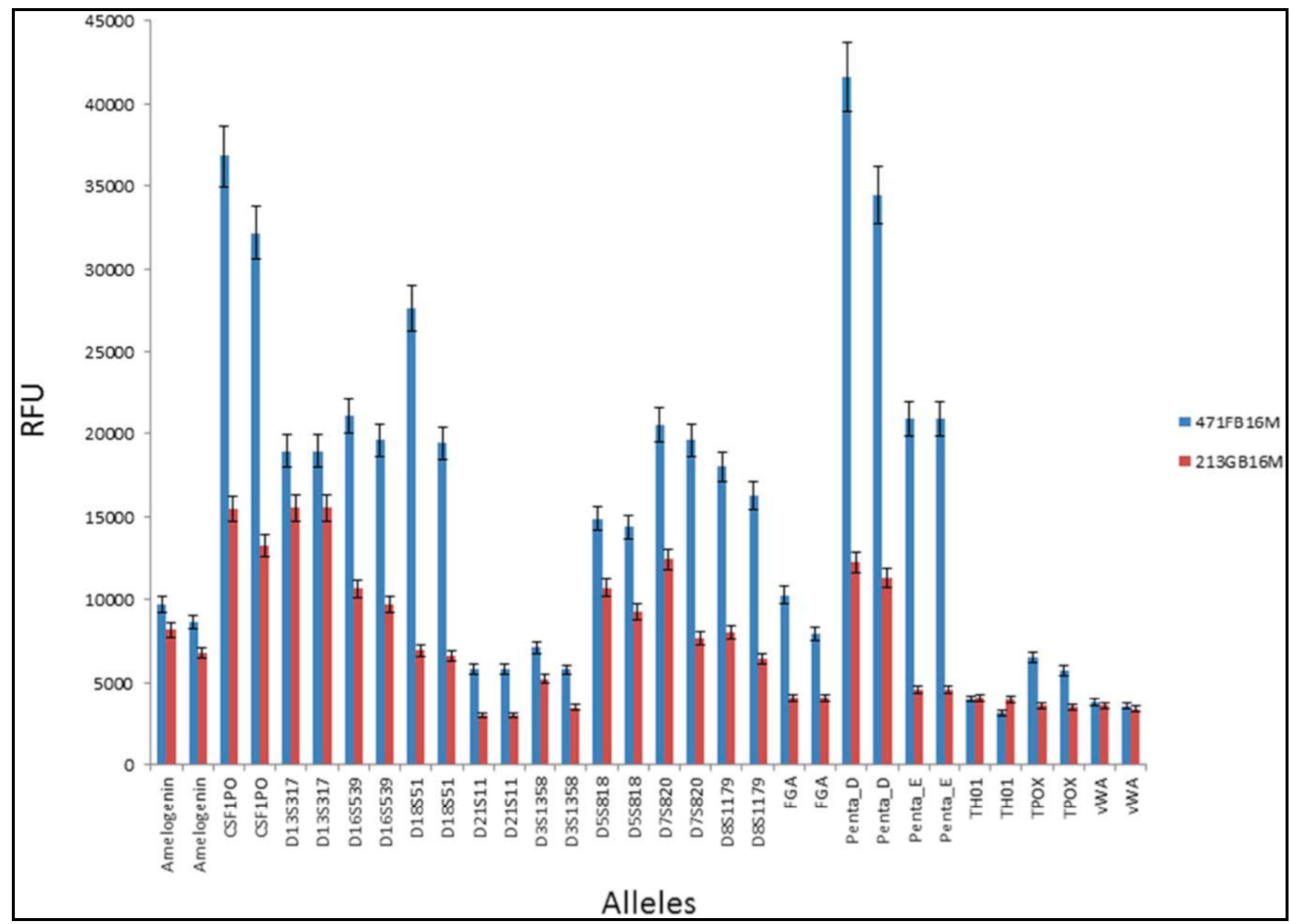

Fig. (3). Florescent intensities of frozen blood from subject $\# 471$ (417FB16M) and florescent intensities of GenPlate ${ }^{\mathrm{TM}}$ blood from subject \#213 (213GB16M) after 16 months storage.

\subsection{Use of DNA from GenPlates ${ }^{\mathrm{TM}}$ as Reference for Case Identification}

Considering that there is interest to use DNA from GB as reference for case identification we used the PowerPlex 16 STR profile from FB as cases and the profile from GB as references. All the 6 cases were correctly identified by linkage to data from the 24 reference samples. Since the PowerPlex 16 system is validated for human identification, our study suggests that GenPlates ${ }^{\mathrm{TM}}$ DNA can be used as the source of reference DNA for human identification. Although all STR's were correctly assigned with DNA from GB, the signal intensity for GB DNA was lower than the signal intensity for FB at most of the loci. This finding is of interest because equal amounts of DNA were used for the PCR and genotyping analysis steps, thus the differences observed in signal intensity is likely not due to difference in total DNA input. It is likely that there are compounds that leach out of the GenPlates ${ }^{\mathrm{TM}}$ paper that affect PCR efficiency and/or quench the fluorescence intensity. Nonetheless, all signal intensities were above the established threshold (200RFU) and the lower intensity observed did not affect allele calls and identification of the 6 cases.

\section{CONCLUSION}

This paper provides data directly comparing the yield and quality of DNA isolated from blood collected in
GenPlates $^{\mathrm{TM}}$, transported and stored at room temperature with those from frozen blood. The purpose of the study was to determine how yield and genotyping quality may be affected by dry-state ambient temperature transport of DNA

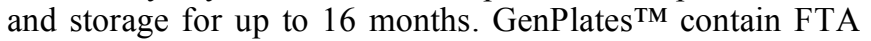
paper which is chemically treated to inactivate bacteria and viruses; and it offers a convenient microtitre plate-based platform for collection, transport and storage of blood. In this study $\sim 0.08 \mathrm{ug} / \mathrm{ul}$ were recovered after 16 months drystate ambient temperature storage. These values are $\sim 5 \%$ higher than the amount recovered from frozen blood, for the same period.

The key factors that will influence the choice of a method for transport and developing a dry-state room temperature reference DNA bank for forensics and other applications includes the: a) amount of DNA recoverable after storage, b) quality of the recovered DNA, c) ease of DNA recovery and d) overall cost of the collection and storage. This study which focused on suitability of using GenPlates ${ }^{\mathrm{TM}}$ to collect and transport blood, as well as yield and quality of isolated DNA clearly demonstrates that blood spotted into GenPlates $^{\mathrm{TM}}$ and stored for up to 16 months produces DNA that is comparable in yield and quality to DNA from blood stored at $-80^{\circ} \mathrm{C}$.

Recently, the performance of three products developed for dry-state ambient temperature transport and storage of 
purified DNA was reported. Specifically, GenTegra ${ }^{\mathrm{TM}}$ (GenVault Corp), Qiasafe ${ }^{\mathrm{TM}}$ (Qiagen Inc) and SampleMatrix ${ }^{\mathrm{TM}}$ (Biomatrica) developed for dry-state ambient storage of purified DNA was compared to frozen storage. It was determined that the yield and quality of DNA stored in GenTegra' ${ }^{\mathrm{TM}}$, Qiasafe ${ }^{\mathrm{TM}}$ and $-20^{\circ} \mathrm{C}$ [6] and GenTegra $^{\mathrm{TM}}$, SampleMatrix ${ }^{\mathrm{TM}}$ and $-80^{\circ} \mathrm{C}$ [7] were generally comparable. Accelerated shelf-life studies by GenVault and Biomatrica suggest that the quality of purified DNA stored at ambient temperature can be maintained for a minimum of 10 years and 30 years, respectively.

In the present study, even though the signal intensity for GB was consistently lower, the intensity from 1ng of starting DNA was sufficient to correctly identify 6 cases on the basis of PowerPlex 16 STR profile after 16 months dry-state ambient temperature storage. The barcoded microtitre plate format will allow for easy tracking, transport and stacking during transport and storage.

\section{ACKNOWLEDGMENT}

None declared.

\section{CONFLICT OF INTEREST}

None declared.

\section{REFERENCES}

Beckett, S.M.; Laughton, S.J.; Pozza, L.D.; McCowage, G.B.; Marshall, G.; Cohn, R.J., et al. Buccal swabs and treated cards: methodological considerations for molecular epidemiologic studies examining pediatric populations. Am. J. Epid., 2008, 167 (10), 1260-1267.

[2] Smith, L.M.; Burgoyne, L.A. Collecting, archiving and processing DNA from wildlife samples using FTA databasing paper. B.M.C. E. col., 2004, 4, 4

[3] Guthrie, R.; Susi, A. A simple phenylalanine method for detecting phenylketonuria in large populations of newborn infants. Pediatr. 1963, 32, 338-343.

[4] Li, C.C.; Beck, I.A.; Seidel, K.D.; Frenkel, L.M. Persistence of human immunodeficiency virus type 1 subtype B DNA in driedblood samples on FTA filter paper. J. Clin. Microbiol., 2004, 42, 3847-3849.

[5] Vanek, D.; Hradil, R.; Budowle, B. Czech population data on 10 short tandem repeat loci of SGM Plus STR system kit using DNA purified in FTA cards. For. Sci. Int., 2001, 119, 107-108.

[6] Frippiat, C.; Zorbo, S.; Leonard, D.; Marcotte, A.; Chaput, M. Aelbrecht, C.; Noel, F. Evaluation of novel forensic DNA storage methodologies. For. Sci. Int. Genet. 2010, doi:10.1016/j.fsigen.20 10.08.007.

[7] Wan, E.; Akana, M.; Pons, J.; Chen, J.; Musone, S.; Kwok, P.Y.; Liao, W. Green technologies for room temperature nucleic acid storage. Curr. Issues Mol. Biol., 2010, 12, 135-142.

[8] Ensenberger, M.G.; Thompson, J.; Hill, B.; Homick, K.; Kearney, V.; Mayntz-Press, K.A., et al. Developmental validation of the PowerPlex 16 HS System: an improved 16-locus fluorescent STR multiplex. For. Sci. Int. Genet., 2010, 4, 257-264.

[9] Wolfgramm, E.D.; Silva, B.C.; da Costa Aguiar, V.R.; Malta, F.S.; de Castro, A.M.; de Souza Ferreira, A.C., et al. Genetic analysis of 15 autosomal and 12 Y-STR loci in the Espirito Santo State population, Brazil. For. Sci. Int. Genet., 2011, 5, 41-43.

[10] Greenspoon, S.A.; Ban, J.D.; Pablo, L.; Crouse, C.A.; Kist, F.G.; Tomsey, C.S., et al. Validation and implementation of the PowerPlex 16 BIO System STR multiplex for forensic casework. $J$. For. Sci., 2004, 49,71-80. 